\title{
Promoting potential of adipose derived stem cells on peripheral nerve regeneration
}

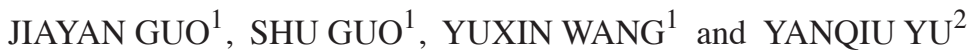 \\ ${ }^{1}$ Department of Plastic Surgery, First Hospital of China Medical University; ${ }^{2}$ Department of \\ Pathophysiology, China Medical University, Shenyang, Liaoning 110001, P.R. China
}

Received June 3, 2016; Accepted July 25, 2017

DOI: $10.3892 / \mathrm{mmr} .2017 .7570$

\begin{abstract}
The ultimate goal of treating peripheral nerve defects is reconstructing continuity of the nerve stumps to regain nerve conduction and functional recovery. Clinically, autologous nerve grafts and Schwann cell (SC) therapy have limitations, such as the need for secondary surgery, sacrifice of another nerve and donor site complication. Adipose derived stem cells (ADSCs) may promise to be ideal alternative cells of SCs. To explore the potential of ADSCs promoting peripheral nerve regeneration, the present study investigated the influences of ADSCs on proliferation and neurotrophic function of SCs using co-culture model in vitro. Western blot analysis, immunocytochemistry, a cell viability assay, reverse transcription-polymerase chain reaction (RT-PCR) and ELISA were applied for examining the interaction of ADSCs and $\mathrm{SCs}$ in a co-culture model in vitro. Western blot analysis and immunocytochemistry demonstrated that protein expression levels of glial filament acidic protein (GFAP) and S100 in ADSCs co-cultured with SCs for 14 days were significantly higher compared with cells cultured alone. Cell viability assay indicated that the cell viability of SCs co-cultured with ADSCs for 3, 4, 5, 6 and 7 days was significantly higher than those cultured alone. RT-PCR showed that expression levels of neurotrophic factors [nerve growth factor (NGF) and glial cell line-derived neurotrophic factor (GDNF)] and extracellular matrix components [fibronectin (FN) and laminin (LN)] in SCs co-cultured with ADSCs for 14 days were significantly higher than those in SCs cultured alone. NGF, GDNF, FN and $\mathrm{LN}$ in the supernatants of co-culture system were significantly higher than cells cultured alone, as ELISA revealed. The results of this study suggested that the transplantation of ADSCs may have a promoting potential to the peripheral nerve regeneration as undifferentiated state.
\end{abstract}

Correspondence to: Dr Shu Guo, Department of Plastic Surgery, First Hospital of China Medical University, 155 Nanjing North Road, Heping, Shenyang, Liaoning 110001, P.R. China

E-mail: guoshu187@sina.com

Key words: adipose derived stem cells, peripheral nerve regeneration, Schwann cells, nerve grafts

\section{Introduction}

The primary aim of treating peripheral nerve defects, which is also one of the major challenges in regenerating medicine, is reconstructing continuity of the nerve stumps to regain nerve conduction and functional recovery. End-to-end suturing is usually applied for nerve lesions without defect or with a short gap; however, nerve grafts are essential to reconstruct long nerve defects. Autologous nerve graft remains the most effective choice, but it is has some limitations, including secondary surgery, painful neuroma formation, scarring and limited donor sites $(1,2)$. In the last few years, cell transplantation, especially for Schwann cells (SCs), has indicated its benefits. SCs serve an important role in peripheral nerve regeneration, and are essential for peripheral nerve development. It has been reported that transplanted cultured SCs could enhance axonal regeneration across nerve gaps (3). Nevertheless, clinical SC therapy is also limited because of secondary surgery, donor site complication and the difficulties of separation, culture and proliferation in vitro. Therefore, it is desirable for alternative cells. Multipotent stem cells, such as neural stem cells and bone marrow stromal cells, which self-renew and multi-potently differentiate, may be the ideal alternative cells. They proliferate rapidly in vitro and differentiate into SC-like cells within specific substrates (4). However, because of several disadvantages, including the morbidity of the donor site, painful procedures and low content, the applications of mulipotent stem cells are restricted.

Recently, adipose derived stem cells (ADSCs), are isolated from adipose tissue, have been identified as having similar phenotype and gene expression profiles to bone marrow stromal cells $(5,6)$. They are multipotent cells that differentiate along several mesenchymal tissue lineages, including adipocytes, osteoblasts, chondrocytes, endothelial cells and cariomyocytes, and express many cytokines and chemokines. ADSCs also have many clinical advantages, including anabundant source, easy and safe accessibility, rapid proliferation and immunological tolerance. Thus, ADSCs may represent alternative cells to SCs. However, the interaction of ADSCs with SCs which exist in nerve stumps remains unknown. It was hypothesized that ADSCs may exert their efficacy by promoting peripheral nerve regeneration not only via differentiation into SC-like cells and direct release of growth factors, chemokines and cytokines, but via indirect modulation of cellular behavior 
of SCs. To test this hypothesis, investigated the influences of ADSCs on proliferation and neurotrophic function of SCs were investigated using co-culture models in vitro.

\section{Materials and methods}

Cell culture. All animal experiments protocols were approved by the Ethics Committee of China Medical University (Shenyang, China) and performed according to China Medical University guidelines. A total of 5male Sprague-Dawley (SD) rats, (age, 8-12 weeks; weight 200-250 g), which were obtained by the Experimental Animal Department of China Medical University (Shenyang, China) under room temperature with free access to food and water, were pre-medicated with Ketalar (50 mg/ml, 0.2 ml/100 g body weight; Jiangsu Hengrui Medicine Co. Ltd., Lianyungang, China), and sacrificed to harvest the inguinal fat pad and dissect the adipose tissue (5). The adipose tissue was digested using $0.1 \%$ collagenase type I at $37^{\circ} \mathrm{C}$ for $30 \mathrm{~min}$. Dulbecco's modified Eagle's medium (DMEM) supplemented with $10 \%$ fetal bovine serum (FBS) (both from Gibco; Thermo Fisher Scientific, Inc., Waltham, MA, USA) was added for neutralization, and the stromal vascular fraction was separated from the floating adipocytes by centrifugation at $300-500 \times \mathrm{g}$ at room temperature for $10 \mathrm{~min}$. Subsequently, the cells in the stromal vascular fraction were resuspended and cultured in DMEM supplemented with $10 \%$ FBS. A total of $24 \mathrm{~h}$ later, the adherent cells were reserved by changing the culture medium, and were passaged three times before used insubsequent experiments.

A total of 6 male SD rats (age, 6 days; weight, 10-14 g), which were obtained by the Experimental Animal Department of China Medical University under room temperature with free access to food and water, were pre-medicated with Ketalar (50 mg/ml, 0.2 ml/100 g body weight; Jiangsu Hengrui Medicine Co. Ltd.), and sacrificed to obtain the sciatic nerves. Briefly, the skin of the hind legs of the rats were cut, the thigh muscles were slit with tweezers to expose the sciatic nerves, which were subsequently removed. The sciatic nerves were sectioned into $2-\mathrm{mm}$ segments and digested using $0.25 \%$ trypsin and $1 \%$ type I collagenase at $37^{\circ} \mathrm{C}$ for $60 \mathrm{~min}$. The suspension was centrifuged at $300-500 \mathrm{x}$ g at room temperature for $10 \mathrm{~min}$ and resuspended in DMEM supplemented with 10\% FBS. After cell adherence, Cytosine Arabinoside (C1768; Sigma-Aldrich; Merck KGaA, Darmstadt, Germany) solution $(10 \mu \mathrm{M})$ was added to the culture medium and cells incubated for $48 \mathrm{~h}$ to remove fibroblasts. DMEM supplemented with forskolin $(2 \mu \mathrm{M})$ and $10 \%$ FBS was then added for $48 \mathrm{~h}$ to promote proliferation. Following this, purified cells were cultured and passaged at $37^{\circ} \mathrm{C}$ in an atmosphere containing $5 \% \mathrm{CO}_{2}$, once the cells reached $80 \%$ confluence.

Adipogenic and osteogenic differentiation of rat ADSCs. To verify the cell multipotent differentiation, rat ADSCs of three passages were used. After they were grown to $80 \%$ confluence, the cells were cultured for 14 days in adipogenic induction medium, consisting of DMEM supplemented with 10\% FBS, isobutyl-methylxanthine $(0.5 \mathrm{mM})$, dexamethasone $(1 \mu \mathrm{M})$, insulin $(10 \mu \mathrm{M})$ and indomethacin $(200 \mu \mathrm{M})$, and cultured for 21 days in osteogenic induction medium, consisting of DMEM supplemented with $10 \% \mathrm{FBS}$, dexamethasone $(0.1 \mu \mathrm{M})$, ascorbate-2-phosphate $(50 \mu \mathrm{M})$ and beta-glycerophosphate (10 mM). Following this, Oil-Red $\mathrm{O}$ and Alizarin Red S staining were used to confirm adipogenic and osteogenic differentiation of the rat ADSCs at room temperature for 14 and 21 days, respectively. Cells were imaged under light microscopes (Nikon Corporation, Toyko, Japan).

Flow cytometry. Rat ADSCs of three passages were washed three times with phosphate-buffed saline (PBS) after trypsinization. The cells were incubated with fluorescein isothiocyanate (FITC)-conjugated primary antibodies, CD29, CD44, CD45 and CD11, away from light at room temperature for $20 \mathrm{~min}$. After the cells were washed with PBS, flow cytometry (FACS Asia; BD Biosciences, Franklin Lakes, NJ, USA) was used to analyze the samples, the results were analyzed with WinMDI2.9 (Purdue University Cytometry Laboratories, West Lafayette, IN, USA).

Cell viability assay of ADSCs. Rat ADSCs of three passages were seeded into 96-well plates at a cell density of $2 \times 10^{4} / \mathrm{ml}$, and cultured in DMEM supplemented with $10 \%$ FBS. After $1,2,3,4,5,6$ or 7 days' culture, cells were incubated with $10 \mu \mathrm{l}$ Cell Counting kit-8 solution (Beyotime Institute of Biotechnology, Haimen, China) at $37^{\circ} \mathrm{C}$ for $3 \mathrm{~h}$. The samples were measured spectrophotometrically at a wavelength of $450 \mathrm{~nm}$ with a Microelisa reader (Infinite M200PRO, Tecan Group Ltd., Männedorf, Switzerland).

Immunocytochemistry. Rat ADSCs of three passages were used and three experiments were performed for each sample. ADSCs were fixed in $4 \%$ paraformaldehyde and blocked with 5\%normal goat serum (Gibco; Thermo Fisher Scientific, Inc., Waltham, MA, USA) at $37^{\circ} \mathrm{C}$ for $20 \mathrm{~min}$. Following this, cells were incubated with the following rabbit monoclonal primary antibodies, all purchased from Abcam (Cambridge, UK): Anti-nerve growth factor (NGF; 1:100; cat no. ab52918), anti-glial cell line-derived neurotrophic factor (GDNF; 1:100; cat. no. ab176564), anti-fibronectin (FN; 1:100; cat. no. ab45688) and anti-laminin (LN; 1:100; cat. no. ab133645) overnight at $4^{\circ} \mathrm{C}$. Cells were then incubated with FITC-conjugated goat anti-rabbit secondary antibodies (Origene Technologies, Inc., Beijing, China) at room temperature for $30 \mathrm{~min}$. DAPI was used to label the cell nuclei, and then the cells were examined undera fluorescence microscope (IX 71/DP 70, Olympus Corporation, Tokyo, Japan).

ELISA. According to the manufacturer's protocol, ELISA kits all purchased from Abcam were used to examine the concentrations of NGF (cat. no. ab193736), GDNF (cat. no. ab213901), FN (cat. no. ab108850) and LN (cat. no. ab11973) in the supernatants of cultured medium.

Induction of rat ADSCs into SC-like cells. Rat ADSCs of three passages were cultured in DMEM supplemented with $\beta$-mercaptoethanol ( $1 \mathrm{mM}$ ) for $24 \mathrm{~h}$, and then treated with DMEM supplemented with $10 \%$ FBS and all-trans-retinoic acid $(35 \mathrm{ng} / \mathrm{ml}$ ) for $72 \mathrm{~h}$. Cells were washed with PBS and incubated at $37^{\circ} \mathrm{C}$ in SC-like cell induction medium, which was DMEM supplemented with $10 \% \mathrm{FBS}$, forskolin $(14 \mu \mathrm{M})$, platelet-derived growth factor-AA $(5 \mathrm{ng} / \mathrm{ml})$, basic fibroblast 
growth factor $(10 \mathrm{ng} / \mathrm{ml})$ and recombinant human heregulin- $\beta 1$ (200 ng/ml), for 14 days.

Co-culture of ADSCs and SCs. Rat ADSCs of three passages were seeded into plates at a density of $2 \times 10^{3} / \mathrm{ml}$. Cells were cultured into 6-well plates for RT-PCR, western blotting and ELISA, into 24-well plates for immunocytochemistry, and into 96-well plates for cell viability assays. SCs were seeded into Transwell cell inserts (pore size, $0.4 \mu \mathrm{m}$ ) at a density of $2 \times 10^{3} / \mathrm{ml}$, and then incubated at humidified $37^{\circ} \mathrm{C}$ environment with $5 \% \mathrm{CO}_{2}$ for $72 \mathrm{~h}$ in DMEM supplemented with $10 \% \mathrm{FBS}$. The plate with cell inserts not seeded with ADSCs or SCs served as control.

Immunocytochemistry for ADSCs of the co-culture system. ADSCs of the co-culture system which were seeded on the bottom at a density of $2 \times 10^{3} / \mathrm{ml}$ were used for the experiments. Three experiments were performed for each sample. Primary antibodies against S100 (rabbit monoclonal, 1:100, cat. no. ab52642, Abcam) and glial filament acidic protein (GFAP; rabbit monoclonal, 1:100, cat. no. ab68428, Abcam) were added after cell fixing in $4 \%$ paraformaldehyde and blocking with normal goat serum, and incubated overnight at $4^{\circ} \mathrm{C}$. Cells were then incubated at room temperature for $30 \mathrm{~min}$ with FITC-conjugated goat anti-rabbit secondary antibodies (Origene Technologies, Inc.). DAPI was used to label the cell nuclei at room temperature for $3 \mathrm{~min}$, and then the cells were examined with a fluorescence microscope (IX 71/DP 70, Olympus Corporation).

Western blotting. ADSCs of the co-culture system which were seeded on the bottom were used for the experiments. Proteins were extracted using a Nuclear and Cytoplasmic Protein Extraction Kit (P0028, Beyotime Institute of Biotechnology, Haimen, China) and detected by BCA Protein Assay Kit (P0009, Beyotime Institute of Biotechnology). Protein extracts (50 $\mu \mathrm{g}$ per lane) were resolved using 10\% SDS-PAGE and electrophoretically transferred onto polyvinylidene difluoride (PVDF) membranes. Then PVDF membranes were incubated with PBS containing 5\% nonfat milk and $0.1 \%$ Tween 20 at room temperature for $1 \mathrm{~h}$, and blocked with $2.5 \%$ normal bovine serum at room temperature for $1 \mathrm{~h}$. Following this, the membranes were probed with primary antibodies againstanti-S-100 (rabbit monoclonal; 1:1,000; cat. no. ab52642; Abcam), GFAP (rabbit monoclonal; 1:1,000; cat. no. ab68428; Abcam) and GAPDH (rabbit polyclonal; 1:2,500; cat. no. ab9485; Abcam) at $4^{\circ} \mathrm{C}$ overnight. The membranes were then incubated with a horseradish peroxidase-conjugated secondary antibody (cat. no. A0208; 1:5,000; Beyotime Institute of Biotechnology) at room temperature for $1 \mathrm{~h}$, following three washes with TBS and $0.1 \%$ Tween-20. The immuno blots were detected using Enhanced Chemiluminescence western blotting reagents (Beyotime Institute of Biotechnology) after washing, and the image was scanned with a GS800 Densitometer Scanner.

Cell viability assay of SCs of the co-culture system. SCs of co-culture system were seeded into 96-well plates and cultured in DMEM supplemented with 10\% FBS. After 1-7 days of culture, cells were incubated with $10 \mu \mathrm{l} \mathrm{CCK}-8$ at $37^{\circ} \mathrm{C}$ for $3 \mathrm{~h}$. The samples were measured spectrophotometrically at
Table I. Primer sequencesin reverse transcription-polymerase chain reaction.

\begin{tabular}{ll}
\hline Gene & \multicolumn{1}{c}{ Sequence (5'-3') } \\
\hline NGF-F & CCTCTTCGGACACTCTGGATTT \\
NGF-R & TCCGTGGCTGTGGTCTTATCT \\
GDNF-F & CAGAGGGAAAGGTCGCAGAG \\
GDNF-R & ATCAGTTCCTCCTTGGTTTCGTAG \\
FN-F & AAGCTACCATTCCAGGCCAC \\
FN-R & GCTCATCTCCTTCCTCGCTC \\
LN-F & ACAGATTGGCTAAGACCGCA \\
LN-R & ACATCTCAGGCCCCTTCTCT \\
$\beta-$ actin-F & TCCTGTGGCATCCACGAAACT \\
$\beta-$-actin-R & GGAGCAATGATCTTGATCTTC \\
\hline
\end{tabular}

F, forward; R, reverse; LN, laminin; FN, fibronectin; NGF, nerve growth factor; GDNF, glial cell line-derived neurotrophic factor.

$450 \mathrm{~nm}$ with an Infinite M200PRO Microelisa reader (Infinite M200PRO).

Reverse transcription-polymerase chain reaction ( $R T-P C R)$. Total cellular RNA was isolated from ADSCs using RN easy Kit (cat. no. 15596026; Invitrogen; Thermo Fisher Scientific, Inc.), then a reverse transcription reaction was performed using a Reverse Transcription Kit (cat. no. K1691; Invitrogen; Thermo Fisher Scientific, Inc.). The PCR reaction was performed with the following thermocycling conditions: $94^{\circ} \mathrm{C}$ for $45 \mathrm{sec}, 58^{\circ} \mathrm{C}$ for $35 \mathrm{sec}$ and $72^{\circ} \mathrm{C}$ for $40 \mathrm{sec}$ for a total 30 cycles. The primer sequences for NGF, GDNF, FN and LN and $\beta$-actin used in this study are presented in Table I. Image Pro Plus 7.0 (Media Cybernetics, Inc., Rockville, MD, USA) was used for densitometry.

Statistical analysis. All data are expressed as the mean \pm standard deviation. One-way analysis of variance followed by Tukey's post hoc test was used to analyse the differences between groups. Statistical analyses were conducted with SPSS13.0 (SPSS, Inc., Chicago, IL, USA). P<0.05 was considered to indicate a significant difference.

\section{Results}

Identification of rat ADSCs. Rat ADSCs isolated from adipose tissue were adhered after 3,4 h, and the suspended cells were removed after $24 \mathrm{~h}$. Spindle cells presented proliferation from 3 days and colony formation from 5 days, and were passaged after 7 days when they reached $80-90 \%$ confluence. Rat ADSCs of 3 passages were used for further experiments. Rat ADSCs presented large, flat and mono-layer cells, arranged in bundles or whorls (Fig. 1A). Cell viability assay indicated that there was a lag phase of cell proliferation within $48 \mathrm{~h}$, but expanded rapidly from 3 days and appeared peak at 6 days (Fig. 1B). Flow cytometry demonstrated that rat ADSCs of passage 3 were CD29 and CD44 positive, but CD45 and CD11 negative (Fig. 1C). To verify the cell multipotent differentiation, adipogenic differentiation was verified by Oil-Red $\mathrm{O}$ staining which 
A

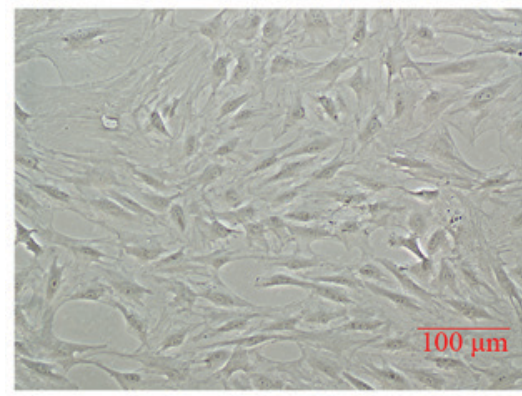

B

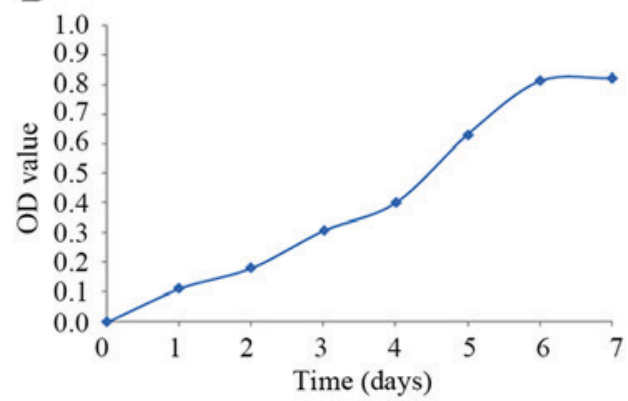

C
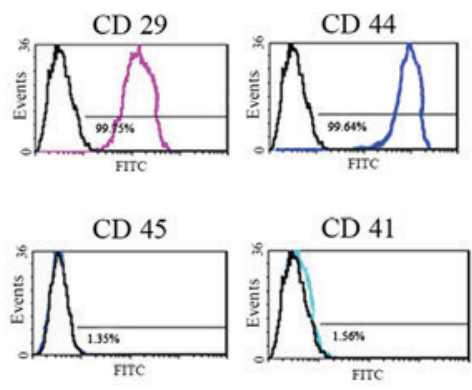

Figure 1. Characteristics of rat ADSCs of 3 passages. (A) Rat ADSCs of 3 passages presented a large, flat and mono-layer shape, arranged in bundles or whorls (B) Cell viability assay indicated that there was a lag phase of cell proliferation within $48 \mathrm{~h}$, but expanded rapidly from 3 days and peaked at 6 days. (C) Flow cytometry demonstrated that rat ADSCs of passage 3 were CD29 and CD44 positive, but CD45 and CD11 negative. CD, cluster of differentiation; OD, optical density; ADSCs, adipose derived stem cells.
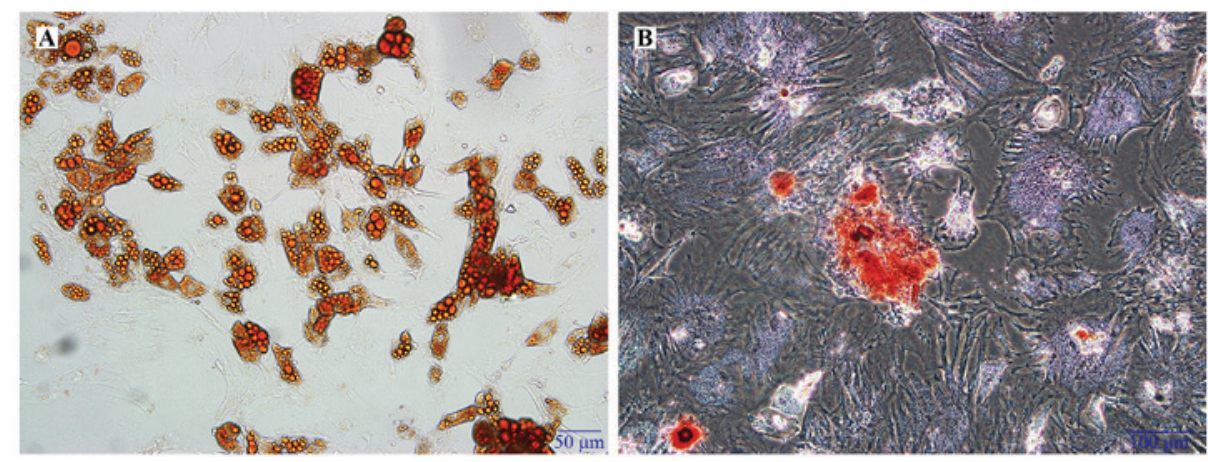

Figure 2. Differentiation of rat ADSCs. When treated with appropriate lineage-specific induction medium, rat ADSCs of 3 passages were successfully differentiated into adipocytes and osteoblasts which were confirmed by (A) Oil-Red O staining and (B) Alizarin Red S staining. ADSCs, adipose derived stem cells.

was demonstrated intracellular lipid droplets (Fig. 2A), while osteogenic differentiation was confirmed by Alizarin Red $\mathrm{S}$ staining, which demonstrated calcium deposits (Fig. 2B).

Secretion function of ADSCs. To determine the secretion function of rat ADSCs, neurotrophic factors such as NGF, GDNF, FN and LN were detected by immunocytochemistry. It demonstrated that ADSCs were positive for NGF, GDNF, FN and LN (Fig. 3A). ELISA revealed that NGF, GDNF, FN and $\mathrm{LN}$ in the supernatants of cultured medium were significantly higher than those cultured alone (Fig. 3B), which was consistent with immunocytochemistry results.

Induction of rat ADSCs into SC-like cells. After incubation in SC-like cell induction medium for 14 days, rat ADSCs of 3 passages which were large and flat were observed as smaller, spindle, bipolar and with long protrusions on two ends, which is similar to SC morphology (Fig. 4A). Also, the SCs-like cells could proceed to proliferation. To determine the SCs-like property, SC markers S100 and GFAP were detected with immunocytochemistry. It demonstrated that S100 and GFAP were positive in SC-like cells (Fig. 4B and C, respectively), but negative in rat ADSCs of 3 passages which were served as the control (data not shown).

Co-culture of ADSCs and SCs. To investigate the influences of ADSCs and SCs on each other, a co-culture model in vitro was applied. Rat ADSCs changed to SCs-like morphology which were smaller, spindle, bipolar and with long protrusions on two ends after 14 days of co-culture (Fig. 5A). Western blot analysis demonstrated that protein expression levels of GFAP and S100 in ADSCs co-cultured with SCs for 14 days were significantly higher than those in ADSCs cultured alone (Fig. 5B). Immunocytochemistry shared consistent results with western blot analysis (Fig. 5C), confirming that rat ADSCs could be induced into SC-like cells in vitro in a co-culture system, compared with the control group.

Cell viability assay indicated that the cell viability of SCs co-cultured with ADSCs for 3, 4, 5, 6 and 7 days was significantly higher than those cultured alone (Fig. 6A). NGF, GDNF, $\mathrm{FN}$ and LN levels in the supernatants of cells in the co-culture system were significantly higher compared with cells cultured alone (Fig. 6B), as ELISA revealed. RT-PCR demonstrated that mRNA expression levels of neurotrophic factors (NGF, GDNF) and extracellular matrix components (FN, LN) in SCs co-cultured with ADSCs for 14 days were significantly higher than those in SCs cultured alone (Fig. 6C). These findings suggested that a higher level of neurotrophic factors and expression levels of extracellular matrix components were present in the co-culture system.

\section{Discussion}

For long nerve defects, the autologous nerve graft is currently the best treatment option due to intrinsic SCs and extracellular matrix proteins. They could provide essential 

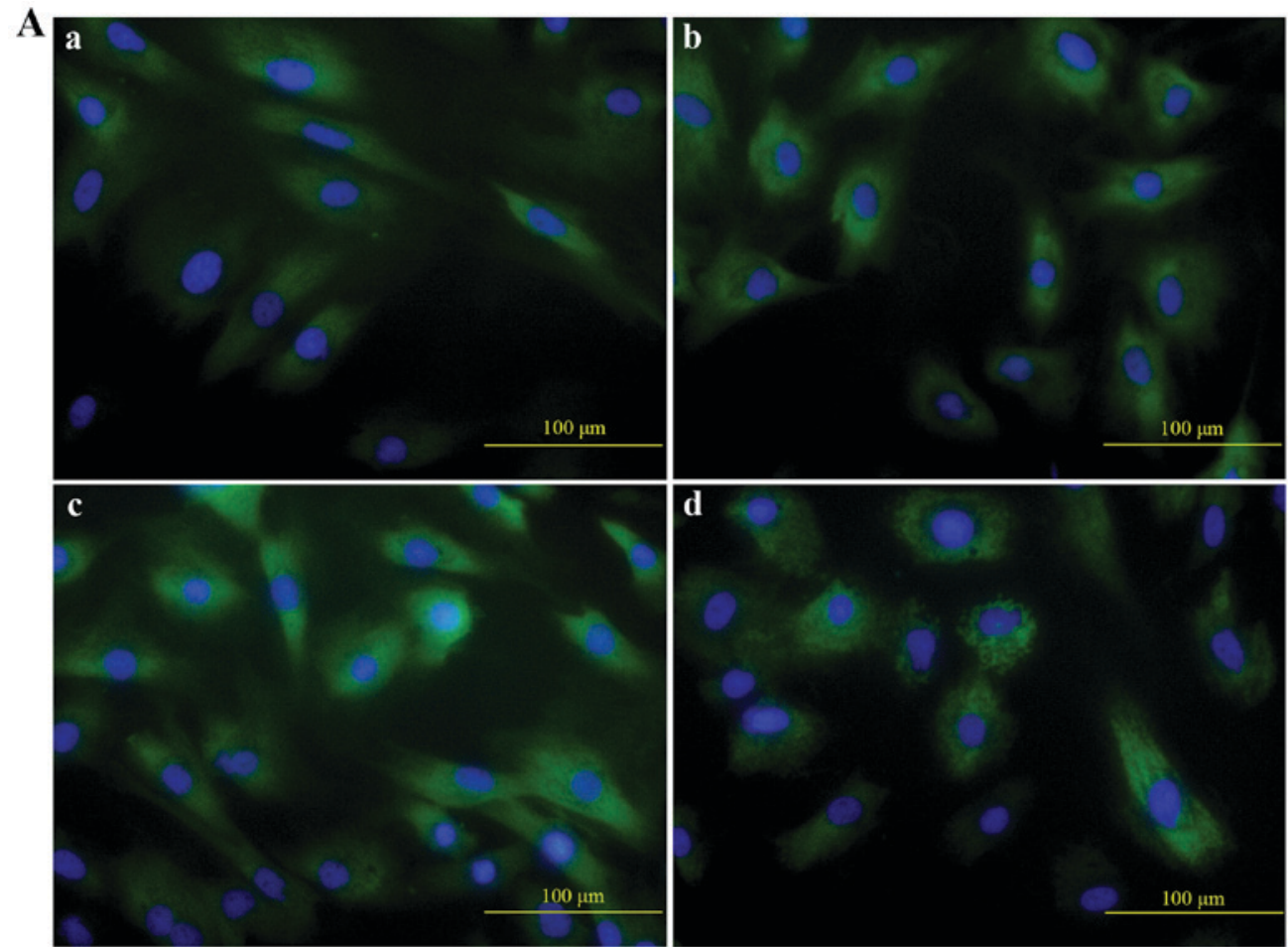

B

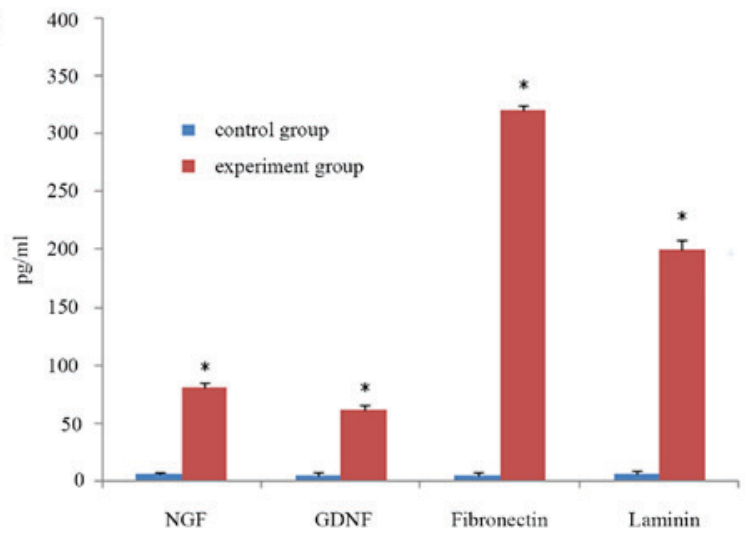

Figure 3. Neurotrophic secretion of rat ADSCs. (A) Immunocytochemistry indicated neurotrophic factors as NGF (panel a), GDNF (panel b), fibronectin (panel c) and laminin (panel d) could be detected in rat ADSCs and supernatants of cultured medium. Green fluorescence indicates the protein of interest, and blue fluorescence indicates nuclei. (B) ELISA results were consistent. Data are expressed as the mean \pm standard deviation. ${ }^{*} \mathrm{P}<0.01 \mathrm{vs}$. control group. ADSCs, adipose derived stem cells.

neurotrophic factors and structural support for peripheral nerve regeneration. Various studies have demonstrated that SCs could enhance axonal regeneration across nerve gaps by means of clearing the degenerated axon, proliferating greatly, secreting neurotrophic factors and providing ideal microenvironment (7-9). However, clinical SC therapy has its limitations because of the need for secondary surgery, sacrifice of another nerve and the donor site complication. Furthermore, it is complextoisolate, culture and SCs. An attractive alternative option of SCs may be multipotent stem cells, as many studies have demonstrated (10-12), such as neural stem cells, hair follicular stem cells and bone marrow stromal cells, which could self-renew and multi-potently differentiate. Although they canproliferate in vitro rapidly and differentiate into SC-like cells within specific substrates, their applications are also restricted because of their disadvantages: The morbidity of donor site, painful procedures and low content.
ADSCs have been used reconstructive medicine in recent years, and they are a more attractive source for tissue engineering compared with other adult stem cells. ADSCs have an abundant source, are easy to isolate from fat tissue and inject immediately post-isolation, are able to self-renew with a high growth rate, leave limited donor site morbidity and possess multi-potent differentiation properties when grown in lineage-specific induction medium (13-15). Previous reports, and the present study, indicated that ADSCs could differentiate into SC-like cells in specific medium, as well as when co-cultured with SCs, and could secrete neurotrophic factors (NGF, GDNF) and extracellular matrix components (FN, LN), which are essential factors for nerve regeneration (3,16-20).

The present study co-cultured ADSCs and SCs, and compared the results with SCs cultured alone. The results demonstrated that following co-culture, more neurotrophic factors and extracellular matrix components were released, and ADSCs could promote SC proliferation and survival and 
A

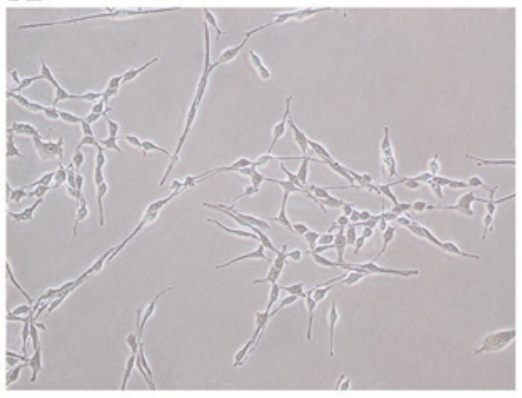

B

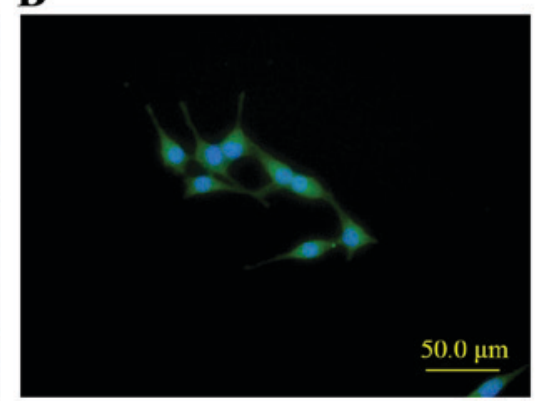

C

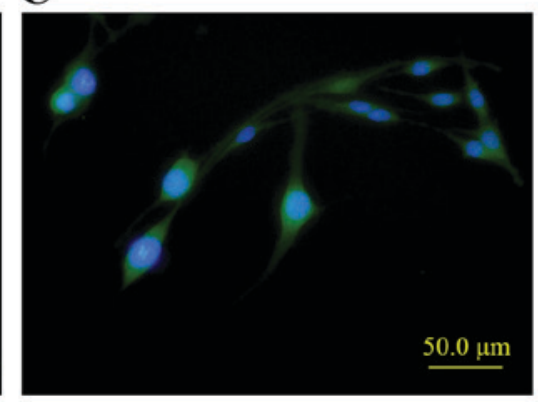

Figure 4. Differentiation of rat ADSCs into SCs-like cells. (A) 14 days after differentiation, rat ADSCs of 3 passages became smaller, had a spindle-like morphology, and were bipolar with long protrusions on two ends (magnification, x100). Immunocytochemistry demonstrated that SC-like cells were positive for (B) S100 and (C) GFAP. Green fluorescence indicates the protein of interest, and blue fluorescence indicates nuclei. GFAP, glial filament acidic protein; SC, Schwann cell; ADSCs, adipose derived stem cells.

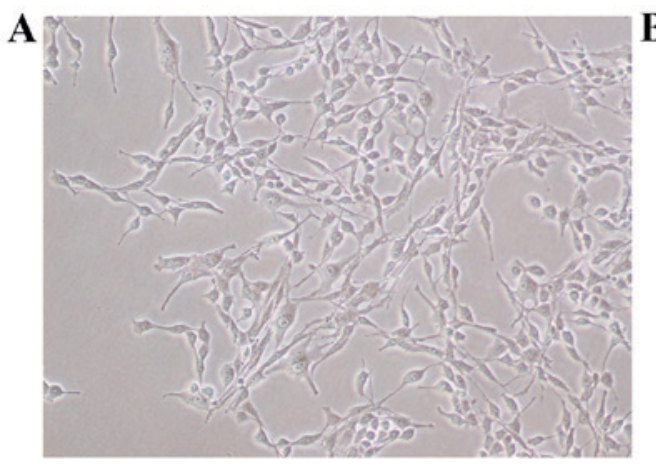

B
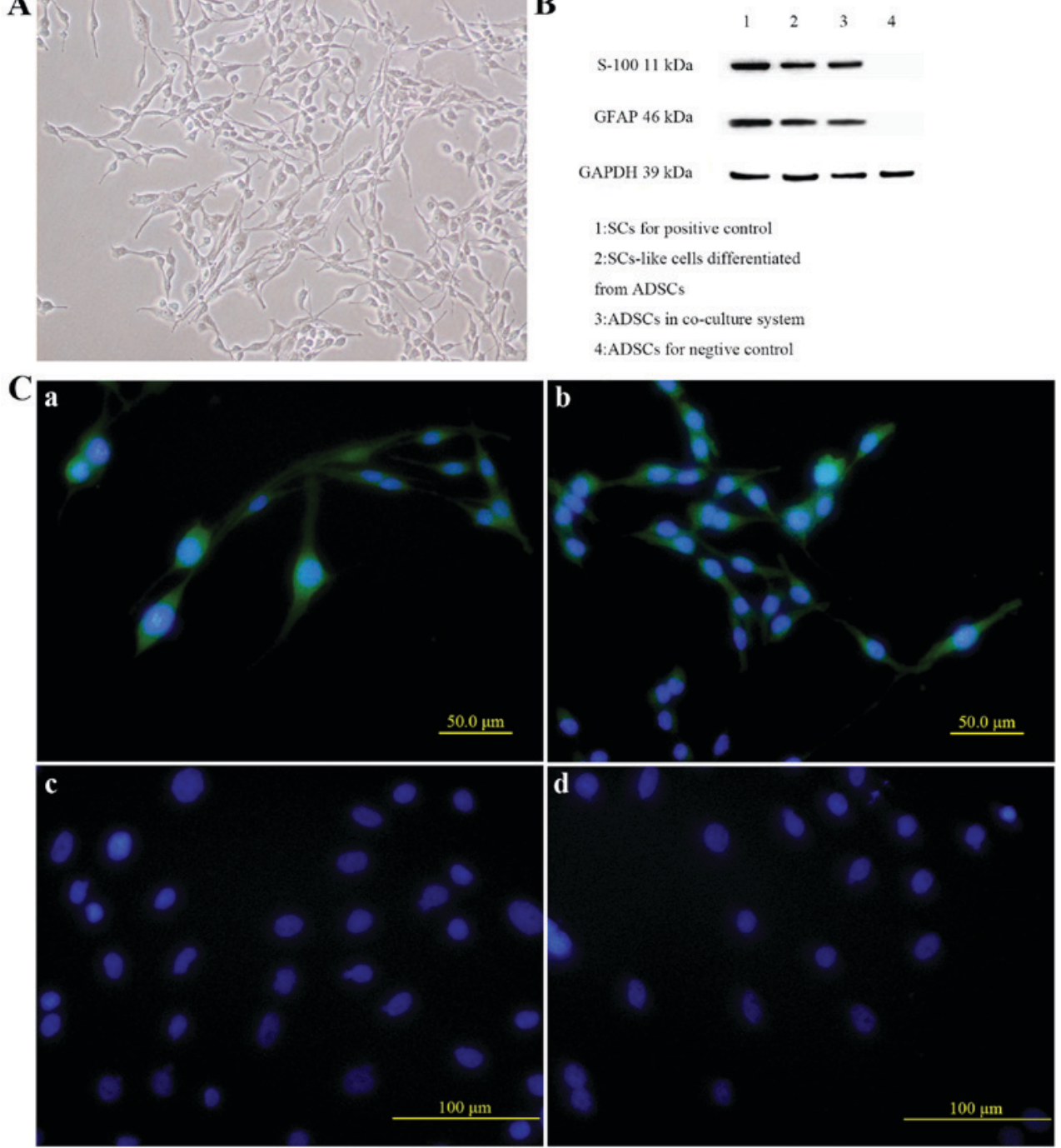

Figure 5. Rat ADSCs in the co-culture system. (A) Rat ADSCs changed to smaller, spindle-like and bipolar morphology, with long protrusions on two ends after 14 days of co-culture (magnification, x100). (B) Western blot analysis demonstrated that protein expression levels of GFAP and S100 in ADSCs co-cultured with SCs for 14 days were significantly higher than those in ADSCs cultured alone. (C) Immunocytochemistry revealed that S100 (panel a) and GFAP (panel b) were positive in SC-like cells and negative in the control group (panel c, panel d). GFAP, glial filament acidic protein; SC, Schwann cell; ADSCs, adipose derived stem cells.

enhance neurotrophic factor expression via released soluble molecules, implicating the neurotrophic active effects and potential to promote peripheral nerve regeneration of ADSCs.
An important factor to be considered is the differentiation state of the applied stem cells. According to the present study, ADSCs could be transplanted into the damaged peripheral 
A

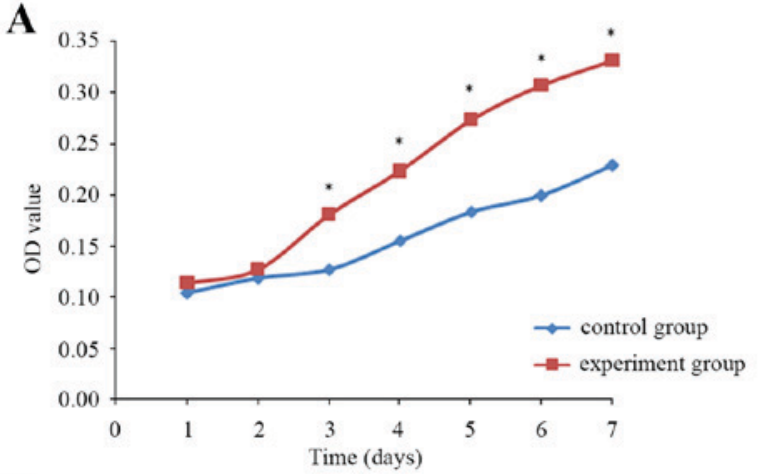

C
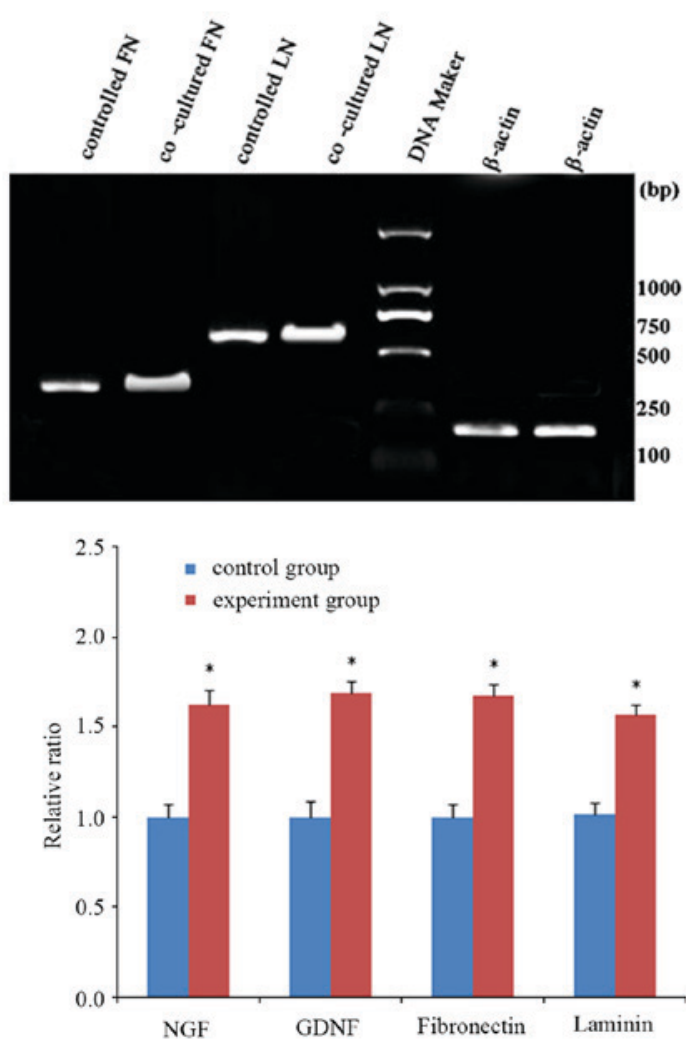

B
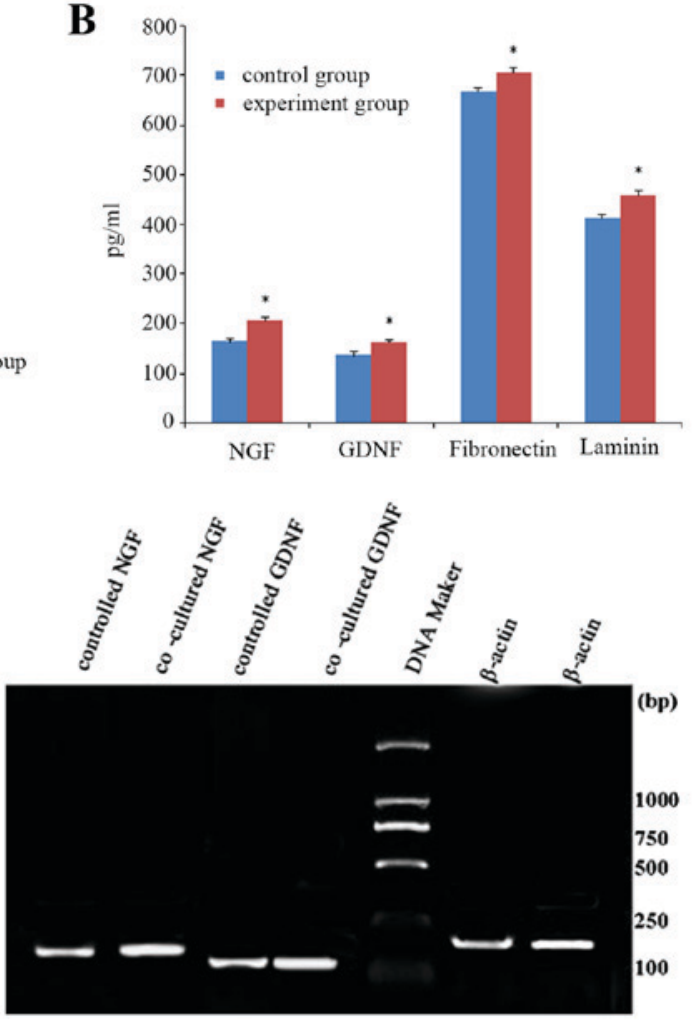

Figure 6. SCs in the co-culture system. (A) Cell viability assay indicated that the cell viability of SCs co-cultured with ADSCs for 3, 4, 5, 6 and 7 days was significantly higher than those cultured alone. (B) Secretion of neurotrophic factors in the co-culture system. NGF, GDNF, FN and LN in the supernatants of SCs in the co-culture system were significantly higher than in SCs cultured alone, as ELISA revealed. (C) Reverse transcription-polymerase chain reaction demonstrated that mRNA expression levels of neurotrophic factors (NGF, GDNF) and extracellular matrix components (FN, LN) in SCs co-cultured with ADSCs for 14 days were significantly higher than those in SCs cultured alone. Data are expressed as the mean \pm standard deviation. "P $<0.01$ vs. control group. FN, fibronectin; LN, laminin; NGF, nerve growth factor; GDNF, glial cell line-derived neurotrophic factor; SC, Schwann cell; ADSCs, adipose derived stem cells; OD, optical density.

nerve directly to promote peripheral nerve regeneration, and avoid the culture and differentiation in vitro. Therefore, the clinical application could be simplified by limiting treatment to one surgical procedure, saving time and costs. Additionally, operating complications are reduced to a minimum. This prediction should be verified by more additional experiments in the future.

Neurotrophic factors and the extracellular matrix serve as trophic and supporting factors that serve a vital role in peripheral nerve development and regeneration, especially for long nerve regeneration, which has been verified by previous studies $(21,22)$. They may stimulate neuritis outgrowth and the proliferation and migration of SCs, and provide a preferable microenvironment for peripheral nerve regeneration. Thus, in addition to SC-like cell differentiation, an alternative possibility for their beneficial effect is the production of neurotrophic factors and extracellular matrix components by co-cultured ADSCs. In the present study, cultured ADSCs were identified to be able to synthesize and release neurotrophic factors such as NGF and GDNF, as well as to produce etxacellular matrix proteins such as FN and LN in vitro, which might partly explain the promoting effects of ADSCs to peripheral nerve regeneration.

Furthermore, ADSCs and SCs have positive effects on each other; ADSCs promote resident SCs proliferating and releasing more neurotrophic factors, while SCs promote ADSCs to differentiate into SC-like cells. The present study demonstrated that even if the transplanted ADSCs couldn't differentiate into 
SC-like cells, they could promote peripheral nerve regeneration by providing a suitable microenvironment for resident SCs of nerve stumps. However, the function of ADSC-differentiated SC-like cells remains controversial.

In conclusion, the results of the present study demonstrated that following co-culture, more neurotrophic factors and extracellular matrix components were released, and ADSCs could promote SC proliferation and survival and enhance neurotrophic factor expression via released soluble molecules. These results suggested that transplantation of undifferentiated ADSCs may have a potential positive effect on peripheral nerve regeneration.

\section{Acknowledgements}

The present study was supported by the National Natural Science Foundation of China (grant no. 51272286).

\section{References}

1. Yoshii S, Oka M, Shima M, Taniguchi A and Akagi M: $30 \mathrm{~mm}$ regeneration of rat sciatic nerve along collagen filaments. Brain Res 949: 202-208, 2002.

2. Hu J, Zhu QT, Liu XL, Xu YB and Zhu JK: Repair of extended peripheral nerve lesions in rhesus monkeys using acellular allogenic nerve grafts implanted with autologous mesenchymal stem cells. Exp Neurol 204: 658-666, 2007.

3. Mantovani C, Terenghi G and Shawcross SG: Isolation of adult stem cells and their differentiation to Schwann cells. Methods Mol Biol 916: 47-57, 2012.

4. Hou SY, Zhang HY, Quan DP, Liu XL and Zhu JK: Tissue-engineered peripheral nerve grafting by differentiated bone marrow stromal cells. Neuroscience 140: 101-110, 2006.

5. Zuk PA, Zhu M, Mizuno H, Huang J, Futrell JW, Katz AJ, Benhaim P, Lorenz HP and Hedrick MH: Multilineage cells from human adipose tissue: Implications for cell based therapies. Tissue Eng 7: 211-228, 2001

6. Heo JS, Choi Y, Kim HS and Kim HO: Comparison of molecular profiles of human mesenchymal stem cells derived from bone marrow, umbilical cord blood, placenta and adipose tissue. Int J Mol Med 37: 115-125, 2016.

7. Fansa $\mathrm{H}$ and Keilhoff G: Comparison of different biogenic matrices seeded with cultured Schwann cells for bridging peripheral nerve defects. Neurol Res 26: 167-173, 2004.

8. Mosahebi A, Woodward B, Wiberg M, Martin R and Terenghi G: Retroviral labeling of Schwann cells: In vitro characterization and in vivo transplantation to improve peripheral nerve regeneration. Glia 34: 8-17, 2001.
9. Rutkowski GE, Miller CA, Jeftinija S and Mallapragada SK: Synergistic effects of micropatterned biodegradable conduits and Schwann cells on sciatic nerve regeneration. J Neural Eng 1: 151-157, 2004.

10. Keilhoff G, Goihl A, Langnäse K, Fansa H and Wolf G: Transdifferentiation of mesenchymal stem cells into Schwann cell-like myelinating cells. Eur J Cell Biol 85: 11-24, 2006.

11. Chen CJ, Ou YC, Liao SL, Chen WY, Chen SY, Wu CW, Wang CC, Wang WY, Huang YS and Hsu SH: Transplantation of bone marrow stromal cells for peripheral nerve repair. Exp Neurol 204: 443-453, 2007.

12. Caddick J, Kingham PJ, Gardiner NJ, Wiberg M and Terenghi G: Phenotypic and functional characteristics of mesenchymal stem cells differentiated along a Schwann cell lineage. Glia 54: 840-849, 2006

13. Strem BM, Hicok KC, Zhu M, Wulur I, Alfonso Z, Schreiber RE, Fraser JK and Hedrick MH: Multipotential differentiation of adipose tissue-derived stem cells. Keio J Med 54: 132-141, 2005.

14. Guilak F, Lott KE, Awad HA, Cao Q, Hicok KC, Fermor B and Gimble JM: Clonal analysis of the differentiation potential of human adipose-derived adult stem cells. J Cell Physiol 206: 229-237, 2006

15. Schäffler A and Büchler C: Concise review: Adipose tissue-derived stromal cells-basic and clinical implications for novel cell-based therapies. Stem Cells 25: 818-827, 2007.

16. Tomita K, Madura T, Sakai Y, Yano K, Terenghi G and Hosokawa K: Glial differentiation of human adipose-derived stem cells: Implications for cell-based transplantation therapy. Neuroscience 236: 55-65, 2013.

17. Gu JH, Ji YH, Dhong ES, Kim DH and Yoon ES: Transplantation of adipose derived stem cells for peripheral nerve regeneration in sciatic nerve defects of the rat. Curr Stem Cell Res Ther 7: 347-355, 2012.

18. Tomita K, Madura T, Mantovani C and Terenghi G: Differentiated adipose-derived stem cells promote myelination and enhance functional recovery in a rat model of chronic denervation. J Neurosci Res 90: 1392-1402, 2012.

19. Sun F,Zhou K, Mi WJ and Qiu JH: Combined use of decellularized allogeneic artery conduits with autologous transdifferentiated adipose-derived stem cells for facial nerve regeneration in rats. Biomaterials 32: 8118-8128, 2011.

20. Kokai LE, Rubin JP and Marra KG: The potential of adipose-derive dadult stem cells as a source of neuronal progenitor cells. Plast Reconstr Surg 116: 1453-1460, 2005.

21. Midha R, Munro CA, Dalton PD, Tator CH and Shoichet MS: Growth factor enhancement of peripheral nerve regeneration through a novel synthetic hydrogel tube. J Neurosurg 99: 555-565, 2003.

22. Mligiliche N, Endo K, Okamoto K, Fujimoto E and Ide C: Extracellular matrix of human amnion manufactured into tubes as conduits for peripheral nerve regeneration. J Biomed Mater Res 63: 591-600, 2002. 